\title{
The effects of adaptation to square-wave gratings as a function of grating orientation*
}

\author{
JOY HIRSCH, BRUCE SCHNEIDER, and MICHAEL VITIELLO \\ Columbia University, New York, New York 10027
}

\begin{abstract}
An adaptation technique was used to measure the selectivity or tuning for grating orientation in the visual system for different orientations of the inspection stimulus. Duration thresholds for grating patterns of constant luminance were determined for 13 test gratings oriented from \pm 5 to 90 deg away from each of five adaptation gratings: $0,22,45,67$, and $90 \mathrm{deg}$. Threshold data obtained for test gratings without prior adaptation indicated higher sensitivity for gratings oriented along the horizontal and vertical axis than along the oblique axis. After adaptation, thresholds increased (sensitivity was reduced) for gratings having similar orientations as the test gratings. However, the functions relating sensitivity reduction to degree of angular disparity between test and adaptation grating did not vary across the five inpsection orientations, i.e., selectivity or tuning for grating orientation appeared to be independent of the orientation of the adapting stimulus.
\end{abstract}

Several studies have suggested the existence of orientation-specific effects in the visual system. It is well known, for example, that visual resolution is poorer for lined stimuli that are oriented obliquely than for those oriented along vertical or horizontal meridia (Taylor, 1963; Campbell, Kulikowski, \& Levinson, 1966). These orientation-specific effects have, in turn, led to the notion of orientation channels, i.e., channels that are "tuned" to detect or process patterns that exhibit a specific orientation in the visual field. The usual method employed to demonstrate the existence of such orientation-selective channels is to examine the effects of a masking grating at a specific orientation on the detection of test gratings having orientations which bracket the masking grating (Campbell \& Kulikowski, 1966; Sekuler, Rubin, \& Cushman, 1968; Gilinsky, 1968; Sekuler, 1965). The general finding has been that the interfering effects of a masking stimulus decreased as the angular disparity between the test and masking grating increased. The rate of reduction in masking which occurs when the disparity between the test and masking grating is increased can be interpreted as a measure of selectivity or tuning of the channel. If the degree of masking declines rapidly, the channel can be said to be highly selective; if the degree of masking declines slowly, the channel can be said to be broadly tuned (with respect to orientation), i.e., less highly selective.

Campbell and Kulikowski (1966), using a simultaneous masking paradigm, have reported results which indicate that the selectivity or tuning of orientation-specific channels is slightly broader for oblique as opposed to vertical or horizontal meridia. The masking grating in their experiment was optically

*This research was supported by National Science Foundation Grant GB 36211. The authors wish to express their appreciation to Norma Graham for her valuable assistance and to Richard Bowen for suggestions concerning the manuscript. We would also like to thank Leonard Matin and Alan Kornheiser for their assistance in calibrating equipment. superimposed upon a test grating of a fixed orientation, and the $O$ adjusted the contrast of the test grating to a just detectable level. However, they explored test gratings only at 90 and $45 \mathrm{deg}$. Moreover, the masking paradigm, where test and masking gratings are presented simultaneously, is only one way in which the selectivity of a channel can be measured. If a specific channel exists, then prolonged exposure to a grating at a particular orientation should reduce the sensitivity of the channel to gratings whose orientations are similar to the adapting grating. Thus, one could view the function relating sensitivity reduction to the disparity between test and adaptation orientation as a measure of the selectivity of the channel.

The present experiment measured orientation selectivity in the visual system with a procedure based on adaptation rather than masking. In Condition 1 of the experiment, sensitivity for a single grating was assessed by determining duration thresholds for detection of gratings at various orientations. In Condition 2, the extent to which adaptation to grating stimuli oriented along the vertical, horizontal, and along three oblique meridia impaired detectability of similar test gratings at different orientations was evaluated. The latter procedure was considered to provide a measure of orientation selectivity, whereas the former provided a measure of sensitivity to the gratings without interference due to prior grating inspection. The results of the baseline procedure (Condition 1) and the adaptation paradigm (Condition 2) showed that while grating sensitivity was maximum at horizontal and vertical meridia, in accord with numerous previous findings, orientational selectivity following grating adaptation was no different for oblique orientations than for vertical and horizontal orientations.

\section{METHOD}

The stimuli were square-wave gratings with equal-area light 
and dark bars and a spatial frequency of 5.2 cycles/deg of visual angle. The gratings were high-contrast positives photographically prepared from ronchi rulings (Edmond Scientific Corporation) and mounted on $4 \times 5$ in. cards. The contrast ratio for the gratings, calculated as $(\mathrm{L} \max -\mathrm{L} \min ) /(\mathrm{L} \max +\mathrm{L} \min )$, was 0.98. Stimuli were presented in a two-channel tachistoscope (Scientific Prototype Model 800F) positioned in metal holders at a viewing distance of $88.26 \mathrm{~cm}$ for each channel. A $5.08-\mathrm{cm}$-diam field stop located in front of the stimulus holder provided a circular viewing field in each channel subtending $3.26 \mathrm{deg}$ of visual angle. Each grating was front illuminated by two General Electric fluorescent lamps, which were coated with a silver-base conductive paint to improve rise time of light output. A Corning glass filter, No. 554 (which did not pass light at wavelengths below $400 \mathrm{~nm}$ and above $540 \mathrm{~nm}$ ) was located in each channel between the grating stimulus and field stop. The filter functioned to further shorten the rise and decay time of luminance in the stimulus display. In order to determine the luminance of the white bars, the luminance of a plain white field made of photographic paper was processed in the same manner as the grid patterns and, as measured with an SEI photometer through the No. 554 filter in both channels of the tachistoscope, was $0.137 \mathrm{~mL}$. A silicon solar cell (Type 2A), in conjunction with a Heathkit amplifier (Model EUW-19B) and a Tektronix oscilloscope (Type 512), was used to monitor the filtered light output throughout the experiment. The rise and decay times were less than $3 \%$ of all stimulus durations employed. Stimulus duration dial readings were calibrated against measurements obtained by a Beckman digital voltmeter (Model 6144) and found to be accurate within $5 \%$.

The first and third authors served as Os for the experiment. Both were emmetropic, with normal visual acuity, and well practiced at the experimental task before final data were collected. At the onset of each experimental session, Os were seated in a lightproof room and allowed to dark-adapt for a 10-min period, followed by $5 \mathrm{~min}$ of viewing the inspection grating. Viewing position was stabilized by the use of a viewing hood attached to the tachistoscope. No fixation point was employed, and the Os were permitted to freely scan the inspection pattern. A trial was signaled when the E said "ready." At the end of each trial, the O verbally responded "yes" or "no," depending on whether he had or had not detected the presence of the test grating. In order to maintain a constant level of adaptation throughout the test period, each trial was initiated by a 5 -sec presentation of the inspection grating, immediately followed by the test grating. The inspection grating was off during the test grating presentation. The next trial began immediately after the test stimulus was terminated. Thus, there was zero delay between the offset of the adaptation stimulus and the onset of the test stimulus and zero delay between the offset of the test stimulus and the onset of the 5-sec adaptation stimulus for the next trial. This adaptation procedure is comparable to that employed by Blakemore and Campbell (1969) to study spatial frequency adaptation. It should be noted that, while the procedure guarantees a constant state of adaptation, it is possible that some of the observed reduction in sensitivity was due to forward and backward masking effects, since the adapting stimulus preceded and followed the test stimulus.

Since the adaptation stimulus was off only for the duration of the test stimulus $(<120 \mathrm{msec})$, its absence was not perceived by either $O$. The effective criterion employed by both Os was the detection of a test grating which appeared to be superimposed on the adaptation grating, even though the adaptation grating was not present. Note that perceptually this is the same criterion employed in studies using the simultaneous masking paradigm (e.g., Campbell \& Kulikowski, 1966), where test and masking gratings were presented simultaneously.

An ascending method of limits procedure was employed such that each test grating was initially presented at a subthreshold stimulus duration and increased each trial until the $O$ indicated that he had detected the test grid. The size of the increments varied from 3 to $10 \mathrm{msec}$, with the larger increments associated with a higher threshold. Following the determination of a threshold for a sequence of trials, the next sequence of trials was initiated and continued as above until the threshold was determined. The results of 10 such trial sequences were averaged in order to determine the duration threshold for a given test grating. The $O$ then readapted for $30 \mathrm{sec}$ to the inspection orientation before the threshold for the next test grating in the session was determined in like manner. In Condition 2, duration thresholds were determined for 13 test gratings for each inspection grating. Test gratings were presented at orientations (relative to the inspection grating orientation) of $\pm 5, \pm 11, \pm 22$, $\pm 45, \pm 67$, and $90 \mathrm{deg}$. The order of test pattern presentation was randomized within each experimental session. Five inspection orientations were employed: $0,22,45,67$, and 90 deg (vertical). In a given session, Os viewed only one inspection orientation, and experimental sessions were separated by at least $24 \mathrm{~h}$ in order to minimize any residual adaptation effects. Since luminance was matched for both channels, it was not possible to discriminate between the onset of the test grating and the offset of the adaptation grating when the two stimuli were similarly oriented. Consequently, test gratings of the same orientation as the adapting stimulus were not employed. In Condition 1, during a single experimental session, baseline thresholds were determined for test gratings without prior inspection of a grating stimulus. In that session, the Os dark-adapted for $10 \mathrm{~min}$ and then light-adapted for $5 \mathrm{~min}$ by viewing a plain white field in the tachistoscope with luminance equal to the white bars of the grating stimulus. The same trial procedure as described above was employed for each threshold determination, using the featureless field as the adaptation stimulus.

Peak luminance rather than space average luminance was chosen to control level of light adaptation in Condition 1, since it would be expected to yield the more conservative estimate of the change in threshold due to prior grating inspection. That is, in Condition 1, adaptation to the slightly higher level of luminance $(0.3 \mathrm{log}$ units) represented by the peak luminance would, if anything, be expected to yield higher baseline thresholds than would be obtained if the space average luminance was employed. A higher baseline threshold would reduce the estimate of the difference in threshold before and after adaptation to the grating, which would result in a more conservative estimate of sensitivity reduction. Therefore, baseline thresholds after adaptation to a uniform field with luminance equal to the peak luminance of the gratings were compared to thresholds obtained following inspection of the grating stimul's.

\section{RESULTS}

Log sensitivity $[\log (1 /$ duration threshold in seconds $)]$ for test gratings determined without prior adaptation to inspection gratings is shown for both Os in Fig. 1. The data depicted clearly indicate that sensitivity to this spatial frequency reaches a maximum at the vertical and horizontal orientations and decreases to a minimum at 45-deg grating orientations. The values ranged from .0157 to $.0183 \mathrm{sec}$ for S J.H., and from .0171 to $.0197 \mathrm{sec}$ for SM.V. In Figs. 2 and 3, sensitivity reduction is plotted against the angular difference between the orientation of the inspection grating and the orientation of the test grating. Log sensitivity reduction was determined by dividing the threshold for a test pattern after adaptation to an inspection grating ( $T$ adapted) by the threshold for that same pattern without 
Fig. 1. Log sensitivity [ $\log (1 /$ duration threshold in seconds)] as a function of the orientation of the grating.

Fig. 2. Log sensitivity reduction [ $\log (T$ adapted/T nonadapted)] as a function of the angular disparity between the orientation of the inspection and test gratings for S J.H.

Fig. 3. Log sensitivity reduction $[\log (T$ adapted/T nonadapted)] as a function of the angular disparity between the orientation of the inspection and test gratings for S M.V.
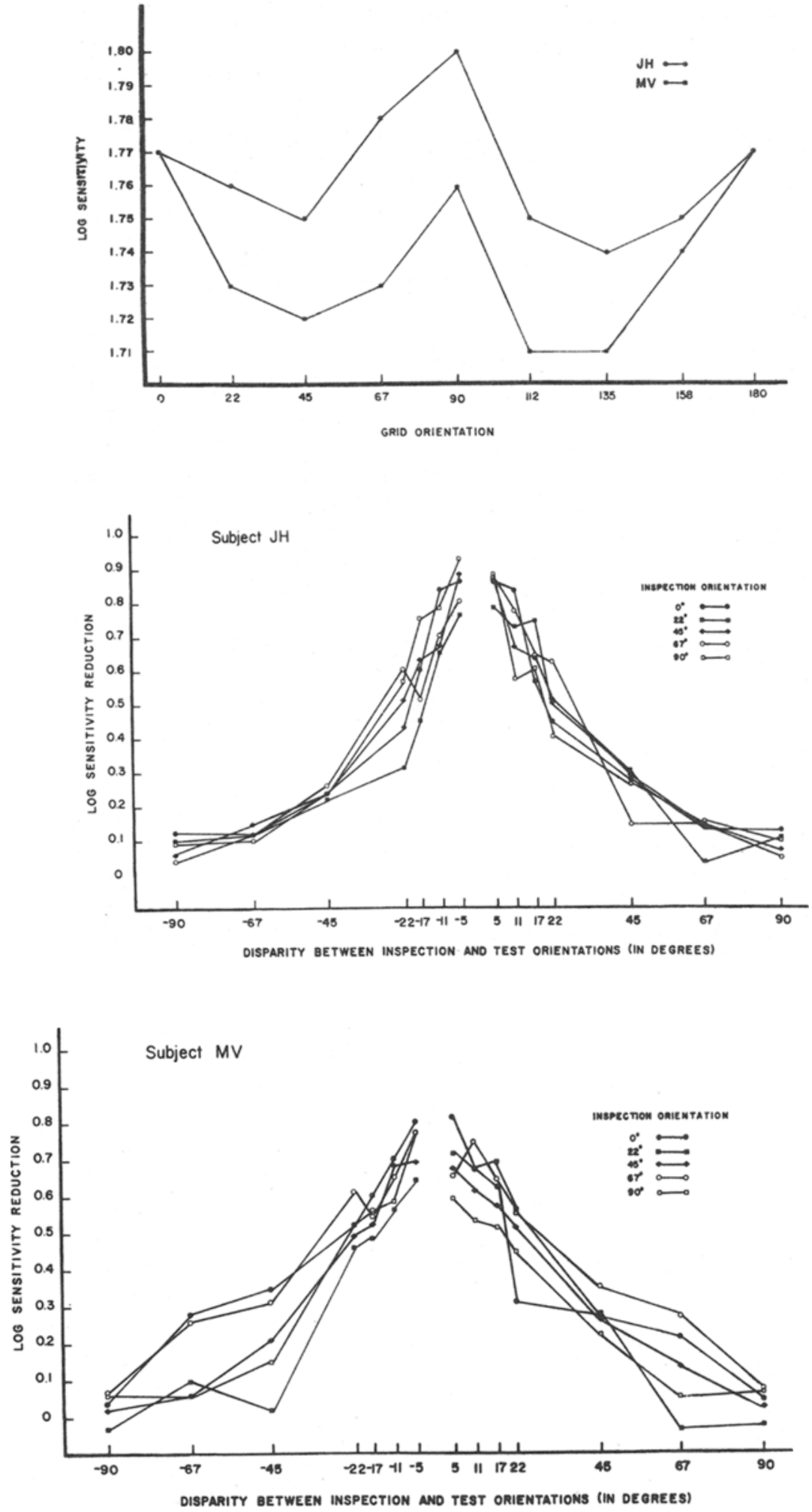

prior adaptation ( $T$ nonadapted) and taking the logarithm of the quotient: $\log$ sensitivity reduction = $\log (\mathrm{T}$ adapted/ $\mathrm{T}$ nonadapted $)$. Since nonadapted thresholds were not determined for all of the test stimuli employed in the adaptation part of the experiment, these thresholds were estimated from Fig. 1 through linear interpolation. The $\log$ sensitivity reduction measure is equivalent to the masking ratio, $\log R$, 
described by Campbell and Kulikowski (1966). The results represented in Figs. 2 and 3 show that sensitivity reduction does not differ systematically for the five inspection orientations. Furthermore, the effects due to adaptation to the inspection grating extended as far as $90 \mathrm{deg}$ angular disparity between the inspection and test grating orientations. Thresholds after adaptation were elevated above the baseline nonadapted thresholds in 10 out of 10 cases for $-67 \mathrm{deg}$ and in 9 out of 10 cases for +67 and $90 \mathrm{deg}$. These values are significant according to a sign test at the $p<.05$ level.

\section{DISCUSSION}

The data of Condition 1 of the experiment are in agreement with the well-known result that measures of grating sensitivity are higher for stimuli oriented along primary axes, i.e., vertical and horizontal, than for gratings oriented along oblique axes (Taylor, 1963; Campbell, Kulikowski, \& Levinson, 1966). Consistent with the high-contrast masking condition employed by Campbell and Kulikowski (1966), the effects of adaptation extended over a 90-deg angle away from the orientation of the adaptation gratings. However, the results of the Condition 2 adaptation procedure fail to confirm the finding obtained by Campbell and Kulikowski, that orientation selectivity is greater for stimuli oriented along a vertical axis than for stimuli oriented along an oblique axis. In the present experiment, selectivity tuning for grating orientation appeared to be independent of the orientation of the adapting stimulus.

Differences in the respective experimental procedures may account for the observed discrepancy in the data. It may be that the slightly broader tuning observed by Campbell and Kulikowski for their oblique orientation might be obscured in our experiment due to greater variability. Second, the sensitivity reduction occasioned by presenting an interfering grating for short simultaneous exposures (the masking paradigm) may differ from that which occurs when the interfering grating is presented for a prolonged period of time prior to the presentation of the test grating (adaptation technique). Finally, it may be the case that the extent of the difference in tuning observed between oblique and vertical channels may be dependent on the spatial frequency of the grating employed. Campbell and Kulikowski employed a spatial frequency of 10 cycles/deg. It may be that there is a difference in tuning for oblique and vertical channels for high spatial frequencies (as shown by Campbell and Kulikowski) but no difference in tuning for medium spatial frequencies as indicated in this experiment (spatial frequency $=5.2$ cycles/deg).

\section{REFERENCES}

Blakemore, C., \& Campbell, F. W. On the existence of neurons in the human visual system selectively sensitive to the orientation and size of retinal images. Journal of Physiology, $1969,203,237-260$.

Campbell, F. W., \& Kulikowski, J. J. Orientational selectivity of the human visual system. Journal of Physiology, 1966, 187, 437-445.

Campbell, F. W., Kulikowski, J. J., \& Levinson, J. The effect of orientation on the visual resolution of gratings. Journal of Physiology, 1966, 187, 427-436.

Gilinsky, A. S. Orientation-specific effects of patterns of adapting light on visual acuity. Journal of the Optical Society, $1968,58,13-18$.

Sekuler, R. W. Spatial and temporal determinants of visual backward masking. Journal of Experimental Psychology, $1965,70,401-406$.

Sekuler, R. W., Rubin, E. L., \& Cushman, W. H. Selectivities of human visual mechanisms for direction of movement and contour orientation. Journal of the Optical Society, 1968, 58, 1146-1150.

Taylor, M. M. Visual diserimination and orientation. Journal of the Optical Society, 1963, 53,763-765.

(Received for publication September 24, 1973; revision received November 19, 1973.) 